\title{
Winner of EUROSON 2014 Young Investigator Award, Maarten Kuenen
}

\section{Winning Abstract \\ $\nabla$}

\section{Contrast-ultrasound dispersion} imaging for prostate cancer localization: comparison between imaging and histopathology

\section{Purpose}

Prostate cancer is the most common form of cancer in men in western countries. Nowadays, diagnosis for prostate cancer is based on systematic biopsies and, as a result, radical treatment is often the only viable treatment option. Reliable imaging techniques could provide significant improvements to prostate cancer care by enabling targeted biopsies and focal therapies. Based on a proven correlation between prostate cancer aggressiveness and angiogenesis, several imaging methods based on analysis of microvascular perfusion have been proposed. However, the effects of angiogenesis on perfusion are complex and influenced by opposing factors. As an alternative to perfusion ima-

contrast agents through the microvasculature than by microvascular perfusion. In this study, the CUDI dispersion maps were compared with histopathology data obtained after radical prostatectomy.

\section{Methods}

CUDI is performed after visualizing the passage of an intravenously injected 2.4mL ultrasound-contrast-agent bolus (SonoVue ${ }^{\circledR}$, Bracco) through the prostate by dynamic contrast-enhanced ultrasound imaging. A time-intensity curve (TIC) is obtained at each video pixel. Based on calibration studies, TICs can be interpreted as indicator dilution curves suitable for analysis of the contrast-agent dispersion kinetics. A local, spatiotemporal dispersion analysis is performed by assessment of the spatial similarity among TICs acquired at neighboring pixels [2, 3, 4]. The parametric dispersion map shown in Fig. 1 is based on spatiotemporal correlation analysis [4]. This method was validated by 43 recordings in 24 patients referred for radi-
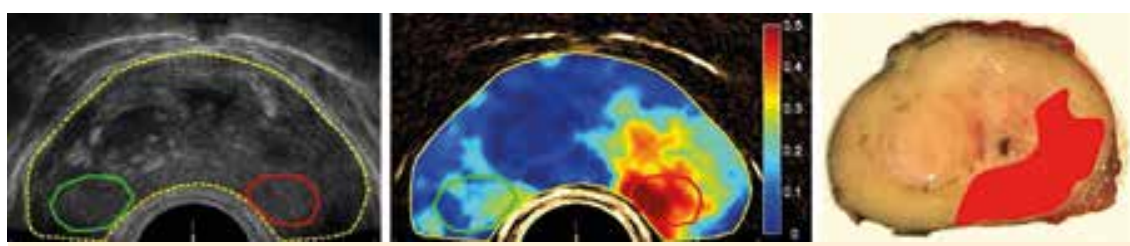

Fig. 1 Ultrasound, dispersion, and histology images with overlaid classification regions.

ging, we have recently proposed contrastultrasound dispersion imaging (CUDI) [1], because typical features of angiogenic microvascular changes, such as density and tortuosity, are better characterized by the intravascular dispersion of ultrasound

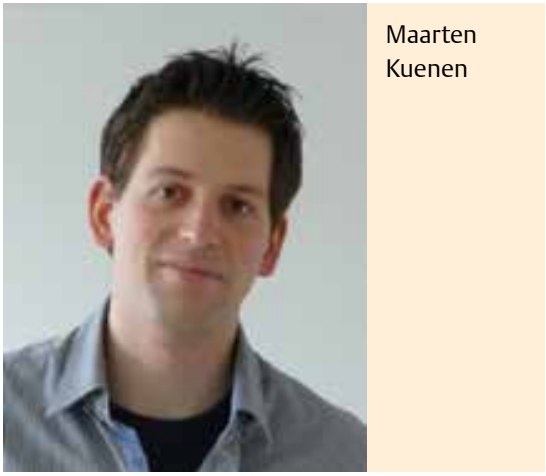

\section{CV}

Maarten Kuenen was born on October 22, 1985 in Venray, The Netherlands. He obtained his M.Sc. degree in electrical engineering from Eindhoven University of Technology in 2009. His thesis concerned the development of quantitative techniques for contrast-enhanced ultrasound imaging for prostate cancer detection. He continued this research in a collaborative project between the Academic Medical Center / University of Amsterdam and the Eindhoven University of Technology under the supervision of Dr. Massimo Mischi. His work resulted in several publications in peer-reviewed academic journals, such as IEEE Transactions on Medical Imaging and Ultrasound in Medicine \& Biology. In particular, for the article "Maximum-likelihood estimation for indicator dilution analysis" published in IEEE Transactions on Biomedical Engineering, Maarten received the IEEE-EMBS Best Paper Award in 2013 from the IEEE-EMBS Benelux Chapter. In March 2014, he received the Ph.D. degree from Eindhoven University of Technology for his thesis "Contrastultrasound dispersion imaging for prostate cancer localization." Since January 2014, Maarten works at Philips Research in Eindhoven. cal prostatectomy at the Academic Medical Center (AMC, Amsterdam, The Netherlands) and the Jeroen Bosch Ziekenhuis (JBZ, 's-Hertogenbosch, The Netherlands) using a Philips iU22 scanner (AMC, 19 patients) and a BK Medical UltraView 800 
scanner (JBZ, 5 patients). The obtained dispersion maps were compared with the histology results on a pixel basis, after selection of two $0.5-\mathrm{cm} 2$ regions of interest based on the histology to represent healthy tissue and cancer, respectively. The classification results were compared to those obtained by perfusion analysis methods described in the literature.

\section{Results}

CUDI by spatiotemporal correlation analysis provided an accurate agreement with histology with sensitivity, specificity, and receiver-operating-characteristic curve area for pixel classification of $77.9 \%, 82.4 \%$, and 0.88 , respectively. These results were over $10 \%$ superior to those obtained by perfusion analysis.

\section{Conclusion}

In conclusion, CUDI has a promising value for localization of prostate cancer. The current results motivate towards a more extensive validation. Future studies may involve investigation of the value of CUDItargeted biopsies, comparison with alternative modalities, such as MRI, and evaluation of CUDI in different forms of cancer, such as breast cancer, that feature similar angiogenic microvascular changes.

M.P.J. Kuenen ${ }^{1,2}$, H.P. Beerlage ${ }^{3}$, J.J.M.C.H. de la Rosette ${ }^{2}$, H. Wijkstra ${ }^{2,1}$, M. Mischi ${ }^{1}$

${ }^{1}$ Dept. of Electrical Engineering, Eindhoven University of Technology, Eindhoven, The Netherlands
${ }^{2}$ Dept. of Urology, Academic Medical Center, University of Amsterdam, Amsterdam, The Netherlands

${ }^{3}$ Dept. of Urology, Jeroen Bosch Ziekenhuis, 's-Hertogenbosch, The Netherlands

References

1 M. P. J. Kuenen, M. Mischi, and H. Wijkstra, "Contrast-ultrasound diffusion imaging for localization of prostate cancer," IEEE Transactions on Medical Imaging, 30(8):14931502, 2011.

2 M. Mischi, M. P. J. Kuenen, and H. Wijkstra, "Angiogenesis imaging by spatiotemporal analysis of ultrasound- contrast-agent dis- persion kinetics," IEEE Transactions on Ultrasonics, Ferroelectrics, and Frequency Control, 59(4):621-629, 2012.

3 M. P. J. Kuenen, T. A. Saidov, H. Wijkstra, and M. Mischi, "Contrast-ultrasound dispersion imaging for prostate cancer localization by improved spatiotemporal similarity analysis," Ultrasound in Medicine \& Biology, 39(9):1631-1641, 2013.

4 M. P. J. Kuenen, T. A. Saidov, H. Wijkstra, J. J. M. C. H. de la Rosette, and M. Mischi, "Spatiotemporal correlation of ultrasound-contrast-agent dilution curves for angiogenesis localization by dispersion imaging," IEEE Transactions on Ultrasonics, Ferroelectrics, and Frequency Control, 60(12):2665-2669, 2013. 